\title{
Effects of combined alkali treatment and clay nanoparticle infusion on thermo-mechanical response of kenaf/PLA biocomposites
}

\author{
M Moyo, K Kanny, TP Mohan
}

\begin{abstract}
Fibre-reinforced biocomposites have inherently low thermo-mechanical properties and hence require some treatments or modifications during the fabrication process in order to enhance these properties. In this work, a combination of alkalization and nanoparticle infusion was used in enhancing thermo-mechanical properties of kenaf fibre-reinforced polylactic acid biocomposites. The biocomposites were made using sodium hydroxide $(\mathrm{NaOH})$ treated kenaf nonwoven mats and polylactic acid infused with clay nanoparticles. Fabrication of the biocomposites was done using the prepreg method and curing at high temperature. Investigation of the thermo-mechanical properties were performed using a thermogravimetric analyser (TGA) and dynamic mechanical analyser (DMA). Results showed that a combination of alkalization and nanoparticle infusion improves the thermal stability of the biocomposites, loss modulus and damping. However, alkalization and nanoparticle infusion decreased the glass transition temperature of the biocomposites. The study shows that combined treatment of biocomposites with sodium hydroxide and clay nanoparticles significantly improves their performance properties. Therefore, this expands the application capabilities of natural fibre-reinforced biocomposites. Best results were obtained by a combination of $\mathrm{NaOH}$ treatment and infusion with $5 \mathrm{wt} \%$ clay nanoparticles.
\end{abstract}

Keywords: Biocomposites, Fibre-reinforced, Thermo-mechanical, Nanoparticles

\section{Introduction}

The work focused on studying the effects of combined alkali treatment and clay nanoparticle infusion on thermo-mechanical properties of kenaf/polylactic acid (PLA) biocomposites. There is a surge of interest in biofibre-reinforced biopolymer composites because they are envisaged as suitable replacements for the conventional synthetic fibre-reinforced polymer composites (Thyavihalli Girijappa et al., 2019; Asyraf et al., 2020; Pickering et al., 2016). Biocomposites are appraised as the next generation materials (Mann et al., 2020; Asyraf et al., 2020). They address pertinent issues of sustainability such as biodegradability, energy conversation, renewability, green manufacturing and reduction of carbon footprint. However, biocomposites are known to have inferior mechanical properties to synthetic fibre-reinforced polymer composites. It is for this reason that biocomposites are currently used for low end applications such as interior parts of automobiles (Siakeng et al., 2018; Rathod et al., 2017; Peças et al., 2018) whereas their applications for exterior parts are very limited. This is due to their inherently poor mechanical properties. Therefore, there is a need to enhance the mechanical properties of the biocomposites so as to expand their application spectrum.

Literature reports techniques used in enhancing properties of natural fibre used in composites production. These techniques include chemical modifications (Cruz and Fangueiro, 2016; Panesar et al., 2017) and other advanced techniques such as biological, plasma and nanoparticle treatments (Cruz and Fangueiro, 2016; Wang et al., 2019). However, little has been done on studying the effects of these techniques on biofibre-reinforced biopolymer composites. Therefore, this work focused on studying thermomechanical properties of kenaf fibre-reinforced PLA biocomposites treated with sodium hydroxide and infused with clay nanoparticles. Chemical modifications bring about better fibre to matrix adhesion than other techniques (Cruz and Fangueiro, 2016; Panesar et al., 2017).

\section{Materials and methods}

\subsection{Materials}

Kenaf nonwoven mats, polylactic acid (PLA) from NatureWorks, USA, Montmorillonite (MMT) cloisite nanoclay from Sigma Aldrich, sodium hydroxide $(\mathrm{NaOH})$, acetone and non-sticking high temperature glass sheet.

\subsection{Treatment with sodium hydroxide}

Kenaf nonwoven mats were treated with $2 \%(\mathrm{~m} / \mathrm{v}) \mathrm{NaOH}$ at room temperature for 48 hours, thereafter the mats were washed using deionised water to remove excess $\mathrm{NaOH}$. Drying of the mats was initially done at room temperature for 24 hours followed by oven drying at $80{ }^{\circ} \mathrm{C}$ for 2 hours.

\subsection{Preparation of nanoclay-doped PLA resin}

Nanoclays were added to a $20(\mathrm{~m} / \mathrm{v})$ PLA acetone solution and homogeneously dispersed using a mechanical stirrer. Nanoclay contents of 0,5 and $7 \mathrm{wt} \%$ were used.

\subsection{Preparation of treated kenaf/PLA biocomposites doped with nanoclay}

$\mathrm{NaOH}$ treated kenaf fibre nonwoven mats were saturated with the nanoclay-doped PLA resin to form prepregs which were dried at room temperature for 2 days. An optimised fibre volume 

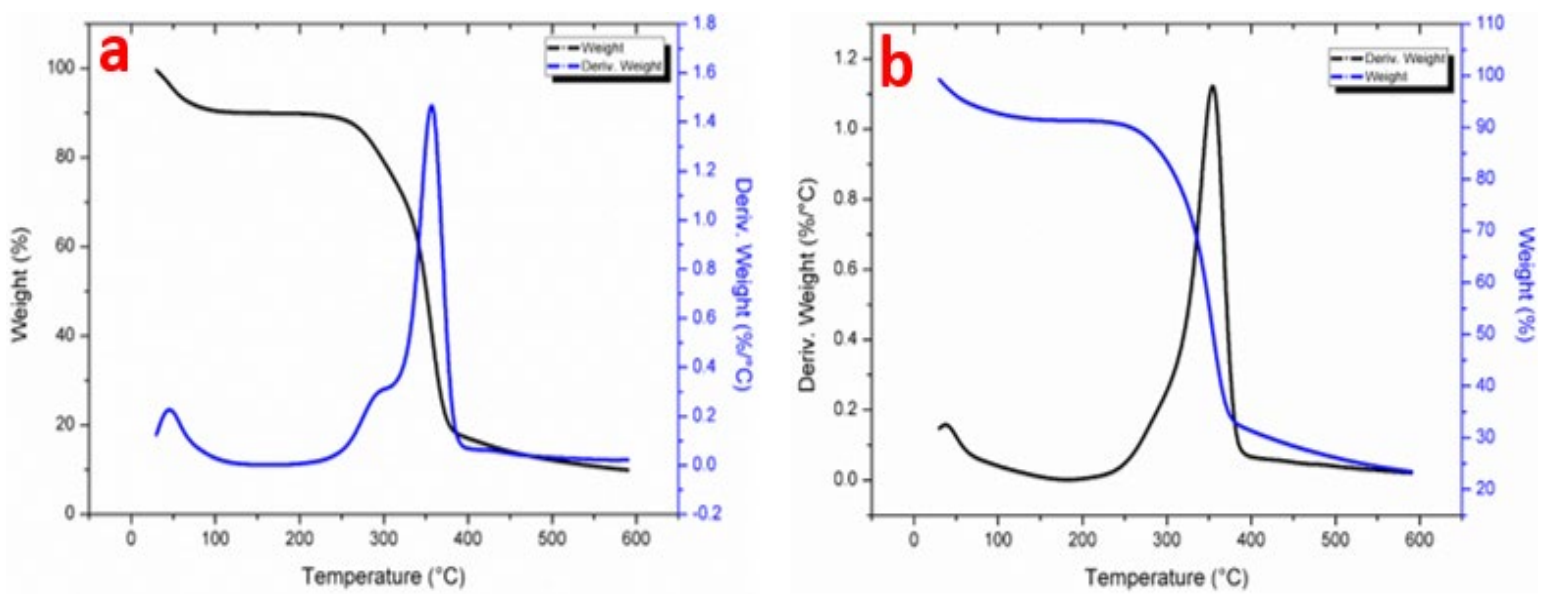

Figure 1: TGA and DTG curves of (a) untreated and (b) $\mathrm{NaOH}$ treated kenaf fibre

fraction of $21 \%$ was used. Curing of prepregs was done in a high temperature oven at $165^{\circ} \mathrm{C}$ for 5 minutes. The non-sticking PTFE high temperature glass sheet was used as a release agent. This preparation method followed the procedure reported by (Moyo et al., 2020).

\subsection{Analysis}

Thermogravimetric Analyser (TGA) was used for analysis of thermal decomposition. and Dynamic Mechanical Analyser (DMA) was used to analyse the dynamic properties of the biocomposites. TGA and DMA were performed using SDT Q600 and DMA Q800, respectively. TGA was done in a nitrogen atmosphere at a heating rate of $10^{\circ} \mathrm{C} / \mathrm{min}$ from $20^{\circ} \mathrm{C}$ to $600^{\circ} \mathrm{C}$. DMA was done using threepoint bending from room temperature to $80^{\circ} \mathrm{C}$.

\section{Results and discussion}

\subsection{Thermogravimetric analysis results}

Figure 1 shows the TGA and DTG curves of untreated (Figure 1a) and treated (Figure 1b) kenaf fibres. Decomposition of volatiles and loss of moisture occurs below $100{ }^{\circ} \mathrm{C}$ and is about $8 \%$. The loss insignificantly contributes to the overall decomposition of the major constituents of natural fibres.

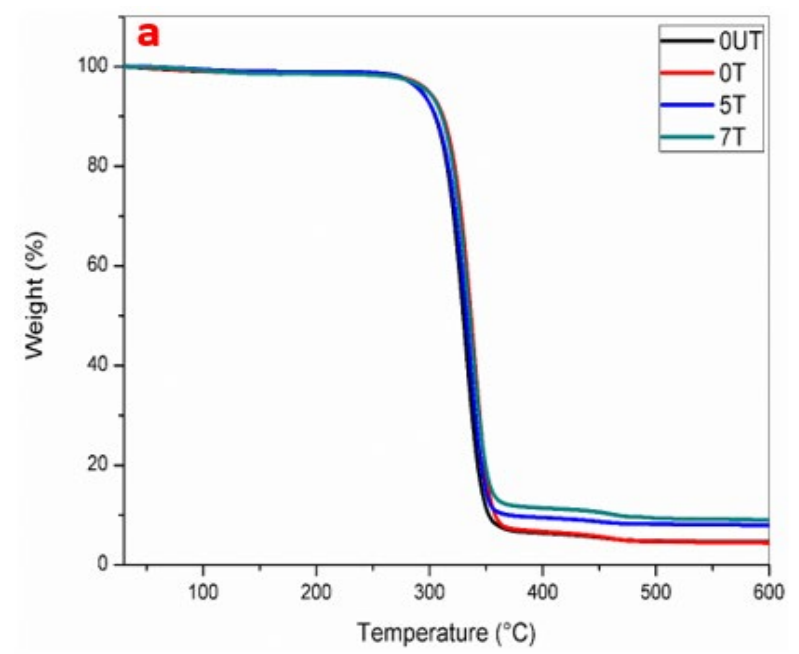

Figure 2: Thermal decomposition of kenaf/PLA biocomposites
Figure 1a shows that a component that decomposed from $250{ }^{\circ} \mathrm{C}$ to $310^{\circ} \mathrm{C}$ is not available in Figure 1b. The decomposed component is hemicellulose since the decomposition range is typical of hemicellulose (Waters et al., 2017). The hemicellulose component which was present in the untreated kenaf fibre was not present in the treated fibre an indication that $\mathrm{NaOH}$ treatment removed the hemicellulose from the kenaf fibre through hydrolysis. Cellulose, which is the major constituent of the kenaf fibre was available after the treatment. In both cases of the untreated and treated fibres, cellulose decomposed from $310{ }^{\circ} \mathrm{C}$ to $390{ }^{\circ} \mathrm{C}$ as shown in both Figures $1 \mathrm{a}$ and $1 \mathrm{~b}$. This indicates that the low concentration of $\mathrm{NaOH}$ used did not cause structural changes that altered the thermal decomposition of the cellulose component of the fibre. After the decomposition of cellulose, the residual mass of the treated and untreated fibre was $33 \%$ and $18 \%$, respectively. At the end of the decomposition (at $600{ }^{\circ} \mathrm{C}$ ), the residual mass of the treated and untreated fibre was $23 \%$ and $9 \%$, respectively, indicating an increase in the thermal stability after $\mathrm{NaOH}$ treatment. This is due to the increase in the crystallinity of the fibre brought about by the removal of the amorphous hemicellulose.

In the following sections, 0UT refers to the biocomposites made using untreated kenaf fibre and PLA resin without nanoclay doping.

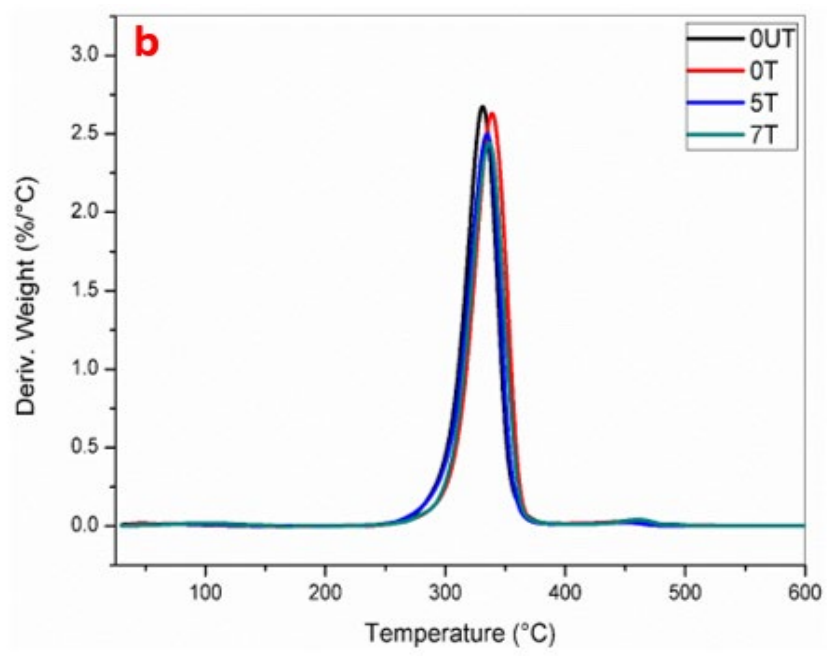


0T, 5T and 7T refer to biocomposites fabricated using $\mathrm{NaOH}$ treated kenaf fibres and nanoclay-doped PLA resin. The nanoclay loading was 0,5 and $7 \mathrm{wt} \%$ for the $0 \mathrm{~T}, 5 \mathrm{~T}$ and $7 \mathrm{~T}$ biocomposites, respectively.

Figure 2 shows the TGA (Figure 2a) and DTG (Figure 2b) curves for the different kenaf/PLA biocomposites. All the biocomposites show a single stage decomposition.

Figure 2a shows that the biocomposites fabricated using $\mathrm{NaOH}$ treated kenaf fibres and nanoclay-doped PLA resin had more residual mass. The residual mass increased with increase in nanoclay loading. Figure $2 \mathrm{~b}$ shows that the peak decomposition temperatures shifted to high temperatures for the treated specimens compared to the OUT specimens. The 0T specimen had the highest peak temperature. These changes show an improvement in the thermal stability.

Table 1 shows a summary of the stages involved in the thermal decomposition process of the treated kenaf/PLA biocomposites and associated mass losses. Table 1 shows that the onset and peak decomposition temperatures increased with increase in the combined treatment of the biocomposites using $\mathrm{NaOH}$ and nanoclay. The onset decomposition temperatures as well as the mass loss increased with increase in nanoclay content. The onset decomposition temperatures improved by $0.4 \%, 0.7 \%$ and $1.5 \%$ for 0T, 5T and 7T biocomposite specimens, respectively. The peak decomposition temperature increased by $2.7 \%, 1.8 \%$ and $2.1 \%$ for 0T, 5T and 7T biocomposite specimens, respectively.

In terms of the residual mass at $377{ }^{\circ} \mathrm{C}$, the improvement was $0.3 \%, 3.3 \%$ and $5.4 \%$ for $0 \mathrm{~T}, 5 \mathrm{~T}$ and $7 \mathrm{~T}$ biocomposite specimens, respectively. These are positive thermal changes indicating an improvement in the thermal stability of the biocomposites. The improvements were better than single method treatments, for instance, acetylation resulted in mass loss of more than $95 \%$ (Chung et al., 2018) whilst the combined treatment in the current work reduced mass loss to $91 \%$. The increase in thermal stability was due to increase in the crystallinity level brought about by treatment of fibres using $\mathrm{NaOH}$ and the thermal barrier effects of the clay nanoparticles. Removal of the hemicellulose component improves the crystallinity of the fibres whilst he thermal barrier effects of the clay nanoparticles lead to reduced heat transfer efficiency in the biocomposite system. The clay nanoparticles reduce the heat transfer rate to the fibres and the PLA matrix thereby delaying the onset of thermal decomposition and also increasing the peak decomposition temperature. This is in agreement with some

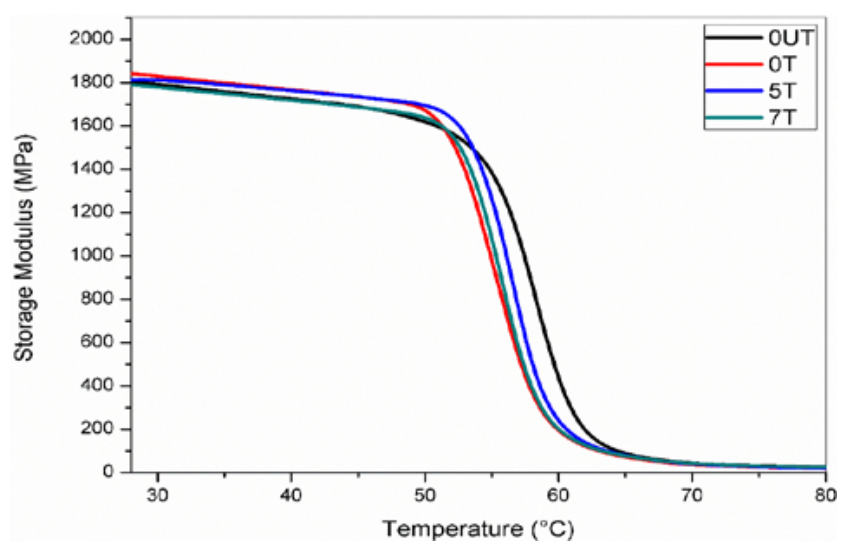

Figure 3: Storage modulus

published literature (Ngo et al., 2016, Moyo et al., 2021). There was no noticeable change in the end decomposition temperature of the biocomposites. This suggests that increase in crystallinity and thermal barrier effects of the clay nanoparticles do not significantly alter the end thermal decomposition temperature of the biocomposites. A similar trend was observed in a study done on kenaf/PLA biocomposites made using untreated kenaf fibre and infused with nanoclay (Moyo et al., 2021). This could mean that the effects on the thermal degradation are less pronounced after the peak decomposition temperature.

\subsection{Storage modulus}

Figure 3 shows that the combined treatments altered the storage modulus. Highest improvement of $2 \%$ was observed on biocomposites fabricated using $\mathrm{NaOH}$ treated fibres without clay infusion due to the increase in crystallinity of the kenaf fibres used. A marginal increase in storage modulus of $0.3 \%$ was observed on biocomposites infused with $5 \mathrm{wt} \%$ nanoclay due to crystallinity effect brought about by the alkali treatment and the effect of the nanoparticle which infused themselves into the biocomposite network structure. Nanoparticles increase the molecular friction and hence increase in storage modulus.

Infusion with $7 \mathrm{wt} \%$ nanoclay resulted in a $0.8 \%$ decrease in storage modulus. The decrease was thought to be due to nanoclay agglomeration effects which reduce molecular friction and hence adversely affecting storage modulus. Microscopic studies on untreated kenaf/PLA biocomposites showed incidences of nanoclay particle agglomeration at the $7 \mathrm{wt} \%$ clay nanoparticle loading (Moyo et al., 2020).

Table 1: Thermal decomposition process of treated kenaf/PLA biocomposites

\begin{tabular}{|c|c|c|c|c|c|c|c|}
\hline \multirow[b]{2}{*}{ Sample } & \multirow{2}{*}{$\begin{array}{l}\mathrm{NaOH} \\
\text { treated }\end{array}$} & \multirow{2}{*}{$\begin{array}{l}\text { Nanoclay } \\
\text { Content }\end{array}$} & \multirow{2}{*}{$\begin{array}{l}\text { Process } \\
\left({ }^{\circ} \mathrm{C}\right) \\
\text { Onset }\end{array}$} & \multicolumn{2}{|c|}{ temperature } & \multirow{2}{*}{$\begin{array}{l}\text { Mass } \\
\text { Loss } \\
\text { (wt.\%) at } \\
377^{\circ} \mathrm{C}\end{array}$} & \multirow{2}{*}{$\begin{array}{l}\text { Mass } \\
\text { Loss } \\
\text { (wt. \%) at } \\
600^{\circ} \mathrm{C}\end{array}$} \\
\hline & & & & Peak & End & & \\
\hline OUT & No & $0 \%$ wt & 267 & 331 & 377 & 93.26 & 95.43 \\
\hline OT & Yes & $0 \%$ wt & 268 & 340 & 377 & 92.95 & 95.58 \\
\hline $5 \mathrm{~T}$ & Yes & $5 \%$ wt & 269 & 337 & 377 & 90.20 & 92.04 \\
\hline $7 \mathrm{~T}$ & Yes & $7 \%$ wt & 271 & 338 & 377 & 88.23 & 90.97 \\
\hline
\end{tabular}




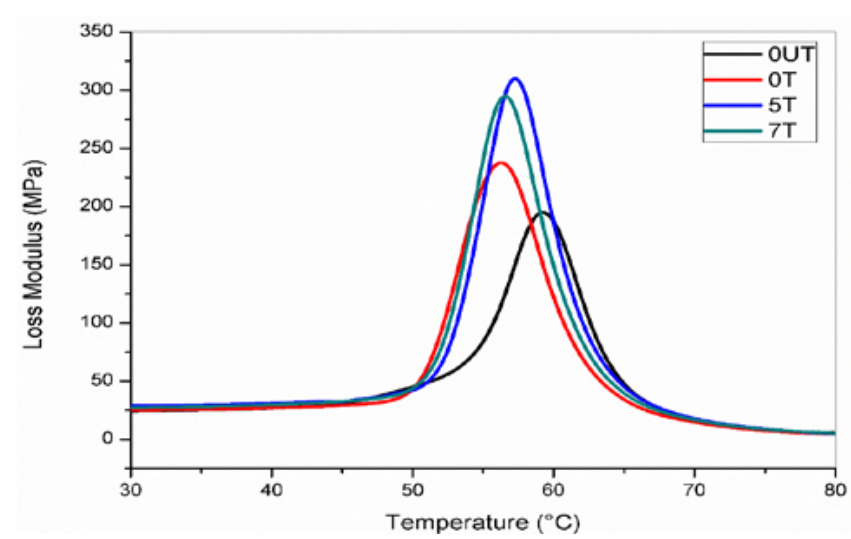

Figure 4: Loss modulus

\subsection{Loss modulus}

Figure 4 shows an increase in the loss modulus of the biocomposites fabricated using combined $\mathrm{NaOH}$ treatment and nanoclay infusion. Biocomposites fabricated using $\mathrm{NaOH}$ treated fibres only showed a $20 \%$ increase in loss modulus and a combination of $\mathrm{NaOH}$ and nanoclay doping increased the loss modulus by $60 \%$ and 51 $\%$ for $5 \mathrm{wt} \%$ and $7 \mathrm{wt} \%$ clay nanoparticle loading, respectively. The increase in loss modulus could be attributed to the improved crystallinity and increased restriction against polymer chain molecular motion due to the nanoclay particles. However, at $7 \mathrm{wt} \%$ nanoclay loading, loss modulus dropped due to clay nanoparticle agglomeration effects.

The temperature corresponding to the peak loss modulus value is the glass transition temperature $(\mathrm{Tg})$ and Figure 4 shows that the $\mathrm{Tg}$ shifted to lower temperature values of $59.3{ }^{\circ} \mathrm{C}, 56.3{ }^{\circ} \mathrm{C}$, $57.3{ }^{\circ} \mathrm{C}$ and 56.6 for 0UT, 0T, 5T and 7T, respectively. The reduction in the $\mathrm{Tg}$ is due to the improvement in the crystallinity level of the biocomposites brought by the alkali treatment and nanoclay infusion.

\subsection{Damping}

The transition peaks shown in Figure 5 show good compatibility of the PLA matrix, kenaf fibres and clay nanoparticles. Figure 5 shows that $\mathrm{NaOH}$ treatment of kenaf fibres did not significantly alter the tandelta value. However, broadening of the curve suggests that $0 \mathrm{~T}$ biocomposites had better damping than 0UT biocomposites. This was due to better interaction between the PLA and the kenaf fibres after the removal of hemicellulose. Addition of $5 \mathrm{wt} \%$ clay nanoparticle resulted in the tandelta values increasing by $25 \%$ showing a significant increase in the damping property of the biocomposites. This was due to increased interaction of the PLA, kenaf fibres and nanoclay as indicated by the shift of the damping peak to a higher temperature. The shift was from $59.8^{\circ} \mathrm{C}$ to $60.3{ }^{\circ} \mathrm{C}$ for $0 \mathrm{~T}$ and $5 \mathrm{~T}$ biocomposites, respectively. However, increase in nanoclay loading to $7 \mathrm{wt} \%$ diminished tandelta by $4.7 \%$ compared to $5 \mathrm{wt} \%$ nanoclay loading. This was due to a reduction in the interaction of the PLA, kenaf fibres and nanoclay as indicated by the shift of the damping peak to a lower temperature. The shift was from $60.3{ }^{\circ} \mathrm{C}$ to $59.7{ }^{\circ} \mathrm{C}$ for $5 \mathrm{~T}$ and $7 \mathrm{~T}$ biocomposites, respectively. Increased nanoclay loading and incidences of agglomeration at 7 $\mathrm{wt} \%$ impose high restrictions against PLA molecular motions.

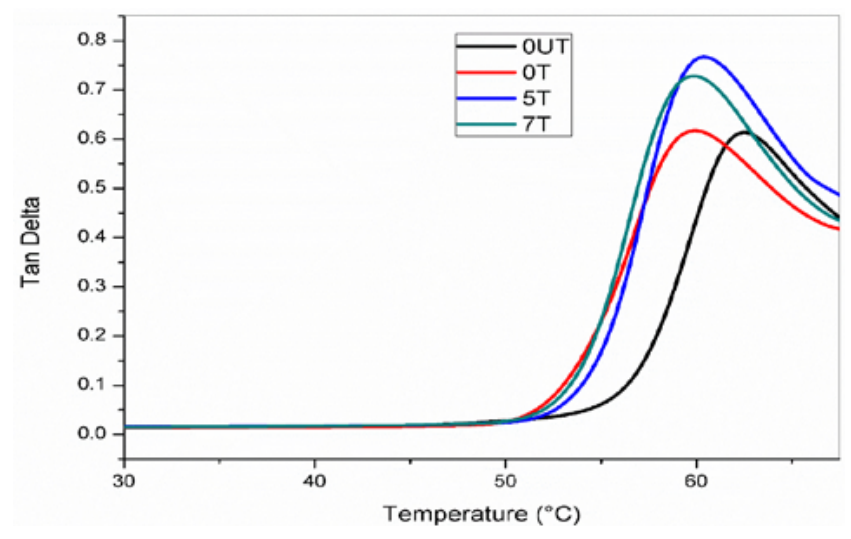

Figure 5: Tan delta plot

The trend observed in this study is in agreement with other published studies that reported an increase in tandelta for composites with clay nanoparticle addition (Chandradass et al., 2007; Mohan and Kanny, 2015; Moyo et al., 2021). However, other studies reported a decrease in tandelta values with the addition of nanoclays for some nanocomposites and other composites (Pothan et al., 2003; Arulmurugan and Venkateshwaran, 2019). The Tg obtained using the tandelta method are $62.4{ }^{\circ} \mathrm{C}, 59.8{ }^{\circ} \mathrm{C}, 60.3{ }^{\circ} \mathrm{C}$ and $59.7^{\circ} \mathrm{C}$ for 0UT, 0T, 5T and 7T biocomposites, respectively. The changes in the $\mathrm{Tg}$ were due to changes in crystallinity and polymer chain molecular motions brought about by combined $\mathrm{NaOH}$ treatment and nanoparticle infusion.

\section{Conclusion}

Combined alkali treatment and clay nanoparticle improved the thermal stability, storage modulus, loss modulus and damping properties of kenaf/PLA biocomposites. The improvements are due to increase in the crystallinity level brought about by $\mathrm{NaOH}$ treatment and clay nanoparticles' thermal barrier effects and effects on molecular friction. The combined alkali treatment and nanoclay infusion lowered the glass transition temperature of the biocomposites. Best results were obtained by a combination of $\mathrm{NaOH}$ treatment and infusion with $5 \mathrm{wt} \%$ clay nanoparticles. $7 \mathrm{wt} \%$ clay content resulted in a drop in properties largely due to nanoclay agglomeration. The combined treatment with $\mathrm{NaOH}$ and nanoclay particles does not have conflicting effects but complimentary effects on the properties of the resultant biocomposites. Other studies have shown that nanoclay particles improve the impact resistance of kenaf/PLA biocomposites in the medium velocity impact range enough for them to cushion against secondary debris (Moyo et al., 2020). Consequently, the synergistic effect of combined treatment would improve both the thermomechanical and impact properties of biocomposites increasing their suitability for exterior and structural applications. Future studies could focus on performing a number of combined treatments of nanoclay and other fibre modification techniques to establish a combination that gives optimum results.

\section{Acknowledgements}

The authors would like to acknowledge the support received from the Durban University of Technology, South Africa. 


\section{Funding}

National Research Foundation of South Africa [Grant UID-105591 and UID-109815] and the Council for Scientific and Industrial Research of South Africa.

\section{Credit authorship contribution statement}

Mufaro Moyo: Conceptualization, analysis and interpretation of data.

Krishnan Kanny: Conceptualization, supervision and critical revisions.

T.P. Mohan: Supervision, review and editing.

\section{References}

1. Arulmurugan S and Venkateshwaran N. (2019) Effect of nanoclay addition and chemical treatment on static and dynamic mechanical analysis of jute fibre composites. Polimeros 29: 1-8.

2. Asyraf MRM, Ishak MR, Sapuan SM, et al. (2020) Potential Application of Green Composites for Cross Arm Component in Transmission Tower: A Brief Review. International Journal of Polymer Science 2020: 1-15.

3. Chandradass J, Kumar MR and Velmurugan R. (2007) Effect of nanoclay addition on vibration properties of glass fibre reinforced vinyl ester composites. Materials Letters 61: 4385-4388.

4. Chung TJ, Park JW, Lee HJ, et al. (2018) The improvement of mechanical properties, thermal stability, and water absorption resistance of an eco-friendly pla/kenaf biocomposite using acetylation. Appl. Sci. 8: 1-13.

5. Cruz J and Fangueiro R. (2016) Surface Modification of Natural Fibers: A Review. Procedia Engineering 155: 285-288.

6. Mann GS, Singh LP, Kumar P, et al. (2020) Green composites: A review of processing technologies and recent applications. Journal of Thermoplastic Composite Materials 33: 1145-1171.

7. Mohan TP and Kanny K. (2015) Nanoclay infused banana fiber and its effects on mechanical and thermal properties of composites. Journal of Composite Materials 50: 1261-1276.
8. Moyo M, Kanny K and Mohan TP. (2021) Thermo-mechanical response of kenaf/PLA biocomposites to clay nanoparticles infusion. Materials Today: Proceedings 38: 609-613.

9. Moyo M, Kanny K and Velmurugan R. (2020) The efficacy of nanoclay loading in the medium velocity impact resistance of kenaf/ PLA biocomposites. Applied Nanoscience 11: 441-453.

10. Ngo TD, Nguyen QT, Nguyen TP, et al. (2016) Effect of nanoclay on thermomechanical properties of epoxy/glass fibre composites. Arab $J$ Sci Eng 41: 1251-1261.

11. Panesar D, Leung R, Sain M, et al. (2017) 2 - The effect of sodium hydroxide surface treatment on the tensile strength and elastic modulus of cellulose nanofiber. In: Savastano Junior H, Fiorelli J and dos Santos SF (eds) Sustainable and Nonconventional Construction Materials using Inorganic Bonded Fiber Composites. Woodhead Publishing, 17-26.

12. Peças P, Carvalho H, Salman H, et al. (2018) Natural Fibre Composites and Their Applications: A Review. Composites Science 2: $1-20$.

13. Pickering KL, Efendy MGA and Le TM. (2016) A review of recent developments in natural fibre composites and their mechanical performance. Composites Part A: Applied Science and Manufacturing 83: 98-112.

14. Pothan LA, Oommen Z and Thomas S. (2003) Dynamic mechanical analysis of banana fiber reinforced polyester composites. Composites Science and Technology 63: 283-293.

15. Rathod VT, Kumar JS and Jain A. (2017) Polymer and ceramic nanocomposites for aerospace applications. Applied Nanoscience 7 519-548.

16. Siakeng R, Jawaid M, Ariffin H, et al. (2018) Natural fiber reinforced polylactic acid composites: A review. Polymer Composites 40: 446-463.

17. Thyavihalli Girijappa YG, Mavinkere Rangappa S Parameswaranpillai J, et al. (2019) Natural Fibers as Sustainable and Renewable Resource for Development of Eco-Friendly Composites: A Comprehensive Review. Frontiers in Materials 6.

18. Wang C, Smith LM, Zhang W, et al. (2019) Reinforcement of Polylactic Acid for Fused Deposition Modeling Process with Nano Particles Treated Bamboo Powder. Polymers 11: 1146.

19. Waters CL, Janupala RR, Mallinson RG, et al. (2017) Staged thermal fractionation for segregation of lignin and cellulose pyrolysis products: An experimental study of residence time and temperature effects Journal of Analytical and Applied Pyrolysis 126: 380-389. 